\title{
Enhancement and Segmentation of Medical Images Using AGCWD and ORACM
}

\author{
https://doi.org/10.3991/ijoe.v16i13.18501 \\ Chenigaram Kalyani \\ Kamala Institute of Technology and Science, Huzurabad, India. \\ Kama Ramudu ${ }^{(凶)}$, Ganta Raghotham Reddy \\ Kakatiya Institute of Technology and Science, Warangal, India \\ ramudukama@gmail.com
}

\begin{abstract}
Images that are obtained in the real world in low contrast are inappropriate for human eyes to read the medical images. Enhancement and segmentation have an important role to play in digital image processing, pattern recognition, and the computer vision. Here, this paper presents an effective way of changing histograms and improving contrast in digital images. Segmentation is done on Adaptive Gamma Correction Weighted Distribution (AGCWD) enhanced images. Histogram equalization is an important technique for contrast enhancement. Nevertheless, modern Histogram Equalization commonly results in unnecessary contrast enhancement, which in turn offers an un-natural presence to the processed image and produces visual artifacts. We present an automated transformation technique that helps boost dimmed image brightness by gamma correction and weighted distribution, commonly known as Adaptive Gamma Correction Weighted Distribution (AGCWD). The contrast enhancement level can be modified using this technique; noise robustness, white or black stretching, and the protection of medium brightness can be easily integrated into the optimization process. Finally, a contrast enhancement algorithm with low complexity is introduced. All the process of enhancement will be done during the process of pre-processing the image. Later, in post-processing, we introduce a specific level set method known as Online Region based Active Contour Model (ORACM) for better segmentation of an enhanced AGCWD image, and it is compared with the traditional level set method.
\end{abstract}

Keywords - Image Enhancement, Histogram Equalization, AGCWD, Image Segmentation, ACM with SBGFRLS, ORACM Level Set-Method

\section{Introduction}

Medical images are a special type of image that can be used to diagnose the disease in patients. There are several methods for obtaining these images, just like computed tomography scan, magnetic resonance imaging and X-ray imaging. Generally, these types of imaging methods result in poor contrast to medical images. The physician's observation is imminent and may lead to a misdiagnosis. Conversely, the attained 
medical images are commonly categorized by a low signal to noise ratio (SNR) in addition to low contrast to noise ratio, along with multiple and discontinuous edges. Various methods of image enhancement have therefore been suggested in the literature [5] to improve the appearance of images for better visual interpretation, understanding, and also for analysis.

Contrast enhancement is a method that adjusts the image histogram to redistribute the pixels between the highest and dimmest portions of the image. In particular, contrast enhancement extends or contrasts the range of intensities in the image, depending on the desired effect. Contrast enhancement is the most widely used technique for image enhancement. In this Image Enhancement, we can observe that there are a lot of side effects while we're going through the process. When there's a low contrast in the image, we're improving the image. They are certain techniques to overcome the shortcoming of this process and they are Histogram Equalization.

A traditional contrast enhancement technique is Histogram equalization [1]. It maps the level of gray based on the input image's probability distribution. But the image produced by this approach will create undesirable effects in the image, and it does not maintain the original brightness of the image. Histogram equalization [2] methodology redistributes probability density. Adaptive Gamma Weighted Distribution Correction technique (AGCWD) is based on the method of modification of histograms. This approach incorporates techniques for gamma correction, as well as techniques for histogram equalization. Gamma correction is a technique for changing the histogram based on transformation, using a variable parameter $\gamma$ (gamma) [8].

The applications of medical images along with object detection as well as image classification have been involved in research progress recently in image segmentation. Image Segmentation [15] plays a crucial part in computer vision and in image processing. A group of the pixels which represent similarly will be in the same region during the segmentation of medical images while the dissimilar pixels are in different regions at the same time.

The main difficulties in the study of images are mainly image segmentation. Many segmentation algorithms [10] in terms of level set method are introduced and generated for various applications. For the most part, we are going over the ORACM Level Set Method (LSM), among various segmentation strategies. A few LSM algorithms had recognized and proven to be successful for both accurate and robust segmentation. The methodology could be applicable for optimized issues of giant proportions, generating prominent arrangements faster than elective methodologies regularly and it also has a low convergence rate in an iterative progression.

This paper had systematized as follows: section 2 describes the literature review; an updated version of the level-set approach is suggested in section 3 as proposed method. Section 4 deals with a thorough description of the experimental tests. Finally, section 5 provides conclusions of this work. 


\section{$2 \quad$ Literature Review}

There are several reasons for poor image contrast, such as quality of capture devices, operator knowledge, or a partially observable environment. Due to poor image quality, it's not an easy task to extract the information that exists in a image. Contrast enhancement rejects the problem by increasing the dynamic array of digital pixel values.

The histogram is a graphic illustration of the distribution of an intensity of the image. In easy terms, it indicates the number of pixels for each of the values of intensity considered. Histogram equalization is achieved by having a transformation function that can be well-defined as "cumulative distribution function"-CDF of a given "probability gray density function"-PDF of a specified image Equalization of histograms is a method used in computer image processing to increase the contrast between images. It is done by allotting the most commonly used intensity standards widely, i.e. by increasing the image intensity range. Typically, this approach improves the total contrast of the image when closely contrast values reflect its accessible data. This allows for higher contrast in places where local contrast is smaller. The image's color histogram reflects the number of pixels for every type of color variation. The histogram equalization couldn't be applied independently to the images' red, green, and the blue components, as this leads to a dramatic variation in the image's color balance.

Some drawbacks refer to existing approaches, like Low Peak SNR, High MSE. Improper photo acquisitions, such as insufficient lighting conditions, may also lead to poor image segmentation. And we need to incorporate an automated technique of transformation to improve contrast and eradicate poor image quality. Adaptive gamma correction via weighted distribution (AGCWD) hybrid HM (Histogram Modification) approach is a combination of transform dependent gamma correction (TGC) along with a traditional histogram equalization (THE) method [8]. This is an automated technique of transformation which increases the dimmed image brightness by gamma correction and by a grayscale probability distribution.

\subsection{Weighted distribution}

Weighted distribution is applied in such a way that high probability image areas shouldn't be over enhanced and the lower-probability image areas must not be less well enhanced, so there should be no loss of important visual information. The input histogram is modified in such a way that greater probabilities or weights are given to less frequent gray rates. Weighted Pdf is given in eq (2). The modified cdf is shown in an eq (3).

\subsection{Gamma correction}

When a value of gamma $\gamma$ is less than 1 then it is treated as gamma encoding, and the process of this compressive power-law non - linearity is called as a gamma compression; conversely, the value of gamma $\gamma$ is greater than 1 then it is a gamma decoding, and the process of an expansive power-law non - linearity is known as a gamma 
expansion [7]. After weighted distribution, Sub-histograms are mapped to the final histogram, later gamma correction is applied [6]. In this method, gamma correction is done with the help of cumulative density function-CDF. Using Gamma correction which is shown in section 3.1.3, the final image in image enhancement is obtained.

Osher-Sethian [16] developed a Level set of methods to match and monitor the interested objects by changing the function of energy instead of the function of a curving. Simultaneously, a system with new ACM's is being developed to overcome the problems related to minimization methods of a classical energy. The Level Set Methods provide robust and resourceful algorithms to search for clarifications to curve equations of evolution. In this paper, we discuss ACM with the model SBGFRLS and ORACM.

\subsection{ACM with SBGFRLS}

Active Contour Model Selective Binary and Gaussian Filtering Regularized Level Set method (ACM with SBGFRLS) involves global and local segmentation properties where Geodesic Active Contour (GAC) and Chane-Vese (C-V) models are to be combined to raise the performance of active segmentation contours of an image. Nevertheless, this procedure does have two big drawbacks. It deforms the active contour initially by using a current level gradient and operates little by little due to a smaller time-stage, boundary balloon leakage, unstable evolution, a low resolution with changes in illumination, and less segmentation precision. Second, it needed a limitation to be adjusted to the input images to play a key role in results. And ORACM can solve the drawbacks described here [11].

\subsection{ORACM}

ORACM is the region-based active contour approach that needs no parameter and less time without altering the precision of the segmentation over conventional ACMs [13].

\section{Proposed Method}

The proposed method of Image Contrast Improvement and the image segmentation is discussed in this section. An algorithm is planned to progress the contrast efficiently and sustain the brightness of input images. Segmentation is performed on the enhanced AGCWD image. The suggested process consists of phases which are shown in the flowchart shown in Figure 1.

\subsection{Image enhancement during pre-processing}

Acquisition of image: Every digital computer, such as cell phones, laptops, and other cameras, can take the image. An image may be grayscale or a color image. In this paper we have taken four standard images. Initially, we verify whether the input image 
is color or a grayscale image. Unless an image is the color image in RGB format, it should be transformed into a grayscale image.

Conversion of RGB image to a gray scale image: Converting an RGB image (red, green, and blue) to a grayscale image, only 8 bits is required to store one pixel of the image. An RGB image is essentially a $A * B * 3$ color pixel array in MATLAB, at which a single-color pixel is the triplet corresponding to the RGB image's red, blue, and green portion at a given spatial position. Similarly, an image of a grayscale is an A*B array whose values are mounted to indicate the intensities.

Image histogram: The algorithm is based on a technique of histogram equalization. Therefore, after the processing of an image, the histogram image is obtained. Histogram of an image is attained by imhist (built-in function).

Adaptive gamma correction via weighted distribution: Rendering to AGCWD process [8], an expression for adaptive gamma correction is expressed as below:

$$
T(1)=1_{\text {MAX }}\left(1 / 1_{\text {MAX }}\right)^{\gamma}=1_{\text {MAX }}\left(1 / 1_{M A X}\right)^{1-C D F(1)}
$$

Weighting Distribution Function is applied as shown below:

$$
P d f_{W}(1)=p d f_{M A X}\left(p d f(1)-p d f_{M I N} / p d f_{M A X}-p d f_{M I N}\right)^{\alpha}
$$

At which $\alpha$ is an adjusted parameter, $p d f_{M A X} \& p d f_{M I N}$ represent maximum and minimum pdf of a statistical histogram. Then modified $c d f$ is expressed as below:

$$
c d f_{w}(1)=\sum_{I=0}^{1_{\max }} P d f w(1) / \sum p d f w
$$

Where the sum of $p d f w$ can be calculated as below:

$$
\sum p d f w=\sum_{I=0}^{1_{\max }} P d f w(1)
$$

Gamma for eqn (1) is deliberated as:

$$
\gamma=1-c d f_{w}(1)
$$

Image enhanced output: Enhanced image is obtained after the completion of gamma correction process. Now, the final enhanced output image is tested with different constraints that will be discussed in the experimental results.

\subsection{Image segmentation during post-processing}

To segment the output of AGCWD image we use conventional Level Set Methods [12]. 
ACM with SBGFRLS: It's a region based active contour method. During the initial stage, user-defined active contour is obtained and then unceasingly modernized through region-based signed pressure function (SPF). And the expression is defined as below:

$$
\operatorname{spf}(I(X, Y))=\frac{I(X, Y)-\frac{C_{1}+C_{2}}{2}}{\operatorname{MAX}\left(\left|I(X, Y)-\frac{C_{1}+C_{2}}{2}\right|\right)}
$$

$C_{1} \& C_{2}$ Represented as

$$
\begin{array}{r}
C_{1}(\phi)=\frac{\int_{\Omega} I(X, Y) H(\phi) d X d Y}{\int_{\Omega} H(\phi) d X d Y} \\
C_{2}(\phi)=\frac{\int_{\Omega} I(X, Y)(1-H(\phi)) d X d Y}{\int_{\Omega}(1-H(\phi)) d X d Y}
\end{array}
$$

Active Contour Model Selective Binary and the Gaussian Filtering Regularized Level set method, uses the Heaviside function with eps $=0$. The signed pressure function role is that it modulates the signs of a pressure force inside as well as outside the interested area. So, contour will shrink outside an object or expand within an object. The same defined definition of degree of variance as shown below is also utilized by the ACM with SBGFRLS:

$$
\frac{\partial \phi}{\partial t}=\operatorname{spf}(I(\mathrm{X})) \alpha|\nabla \phi|
$$

From eq (9) $\alpha$ represented as the constant function and it regulates the speed of a level set up to be changed. The main drawback of the ACM with SBGFRLS is that it must be adjusted according to the image, although it has a significant impact on the outcome. This condition makes it difficult to use the SBGFRLS for simple images, and impossible for video files in real-time. The other downside is slowness. $|\nabla \emptyset|$ denotes as a gradient function of a level set. Using $|\nabla \emptyset|$ the formulation of a level set broadcasts the output of a SPF function only at a limit of the Level Set Method. In this circumstance, only the level set limit is modified, which results in slowness.

ORACM model: ORACM is an active contour-based region which covers the ACM's drawbacks with SBGFRLS. No parameter is required and it takes less time to detect boundary objects. It uses a simple and an effective level collection of modified formulations as follows when compared to the ACM with SBGFRLS: 


$$
\frac{\partial \phi}{\partial X}=H(\operatorname{spf}(I(\mathrm{X}))) \phi(\mathrm{X})
$$

From the above equation, $\mathrm{H}$ (.) represents the function of Heaviside and SPF (.) shown as signed function of the pressure force described in (10).

Compared to the ACM's level-set formulation with SBGFRLS, ORACM doesn't require parameter's to be tuned to the input images to sort the parameter secure. Direct use of the existing level set function reduces measurement time instead of its estimation to curvature. Besides, ORACM uses basic morphological opening as well as closing processes to update the level range smoothly, instead of Gaussian smoothing.

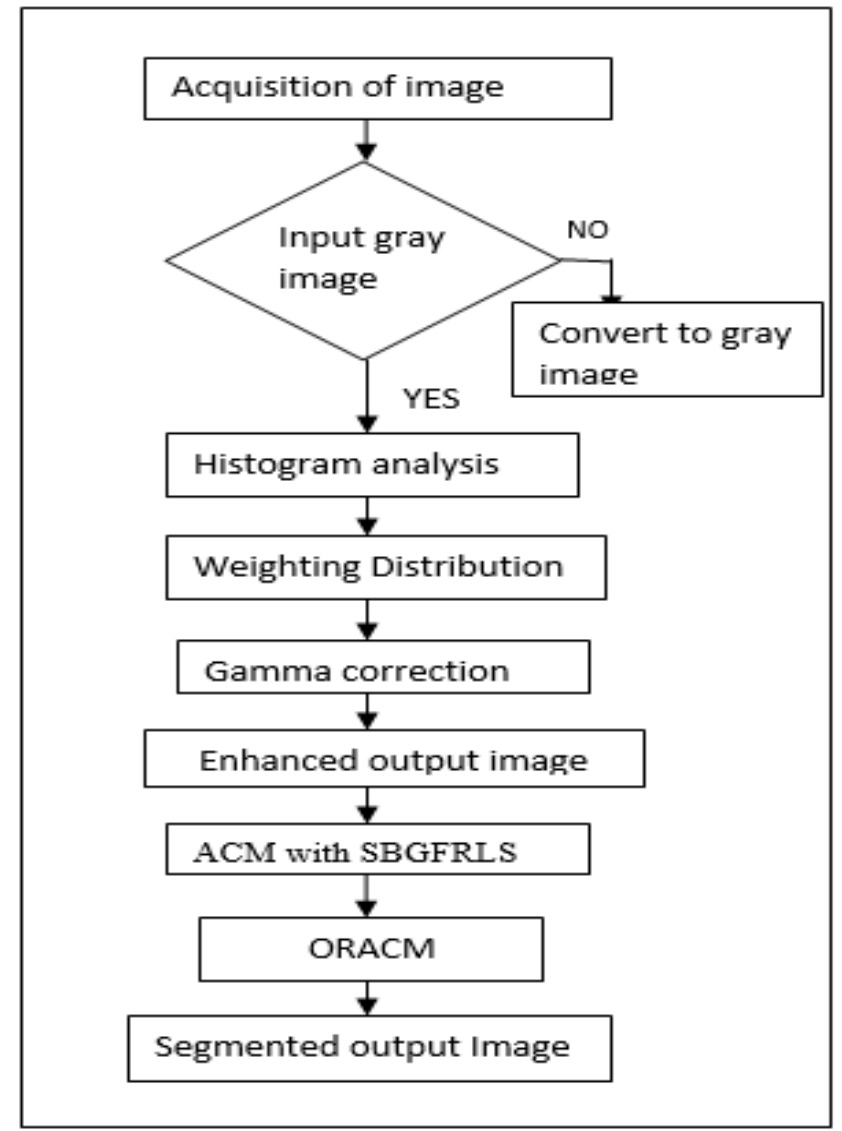

Fig. 1. Flow chart of Proposed Method

Despite a substantial increase in speed and parameter reliability, the use of global information in the SPF feature continues to result in incorrect segmentation, with images having an inhomogeneity of strength. 
Output segmented image: Finally, the segmented image is obtained using ORACM level set method. Now, the output image is tested and compared with the other conventional level set methods by using various constraints which are discussed in the experimental results.

\section{Experimental Results}

Medical images are a distinct kind of image that can be used for the diagnostics of disease in the patients. These types of imaging methods generally result in poor contrast medical images. Results of the proposed methods are contrasted with the techniques such as Histogram equalization (HE), Adaptive gamma correction via weighted distribution-AGCWD, ACM with SBGFRLS, ORACM. Dataset of 4 images are taken into consideration which are used in reference papers previously to relate the results in terms like covered area, less CPU time, and medical images.

Figure 2(a) shows the input MRI image and its histogram output. Similarly, we also have figure 2(b) enhanced output concerning the input image and its histogram output. To have better enhancement during preprocessing we introduce AGCWD shown in figure 3(a). It is clearly shown that, relative to other approaches, the enhanced improvement made by the proposed technique is normal and also stronger. The technique also has improved preservation of original image brightness. On these images performance metrics for example "Peak signal to noise ratio" -PSNR and "Mean Square error" -MSE is measured, and results are given below. As contrasted with traditional ACM methods with SBGFRLS, the proposed level-set method (LSM) ORACM segments the areas or regions correctly and proficiently during post-processing as shown in figure 3 (b) and 3 (c) respectively.

\subsection{Performance measurement}

Output results are calculated using Peak Signal to Noise Ratio (PSNR), Mean Square Error (MSE) for AGCWD as shown in table 1 and table 2.

Peak signal to noise ratio - PSNR: It's a standard assessment of re-constructed excellence of an image, and it is measured in decibel terms $(\mathrm{dB})$ and the equation is expressed through

$$
P S N R=(10 \log 255 / 2 \mathrm{MSE})
$$

From the above equation, the maximum possible value is 255 at which the image signal can achieve. Greater, the PSNR value improves the image being reconstructed.

Mean square error - MSE: Among reference signal and the corrupted signal, difference of mean square is treated as a means square error. The expression for these two signals is shown below: 


$$
M S E=\frac{\sum_{\mathrm{A}, \mathrm{B}}\left[I_{1}(\mathrm{a}, \mathrm{b})-I_{2}(\mathrm{a}, \mathrm{b})\right]^{2}}{\mathrm{~A}^{* \mathrm{~B}}}
$$

Lesser, the MSE value gives the better image quality.

All the algorithms mentioned here are performed with the help of MATLAB R2017a $64 \mathrm{~b}$ in Windows 8 .

Simulation Results of histogram equalization and image enhancement

Input Image and its histogram

Column (a)
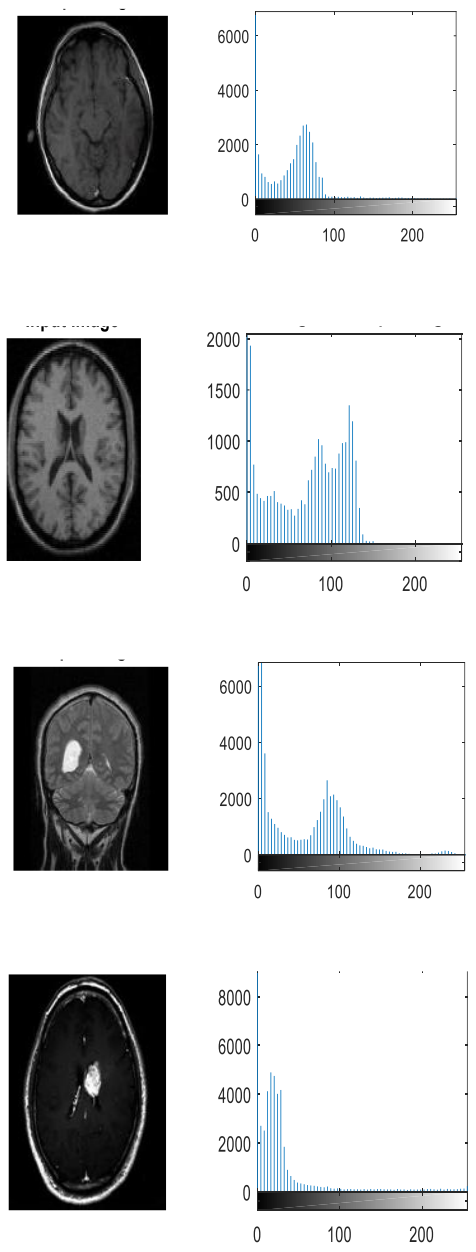
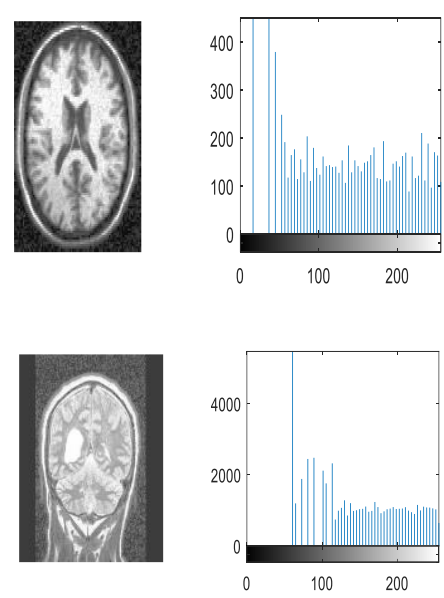

\section{Column (b)}
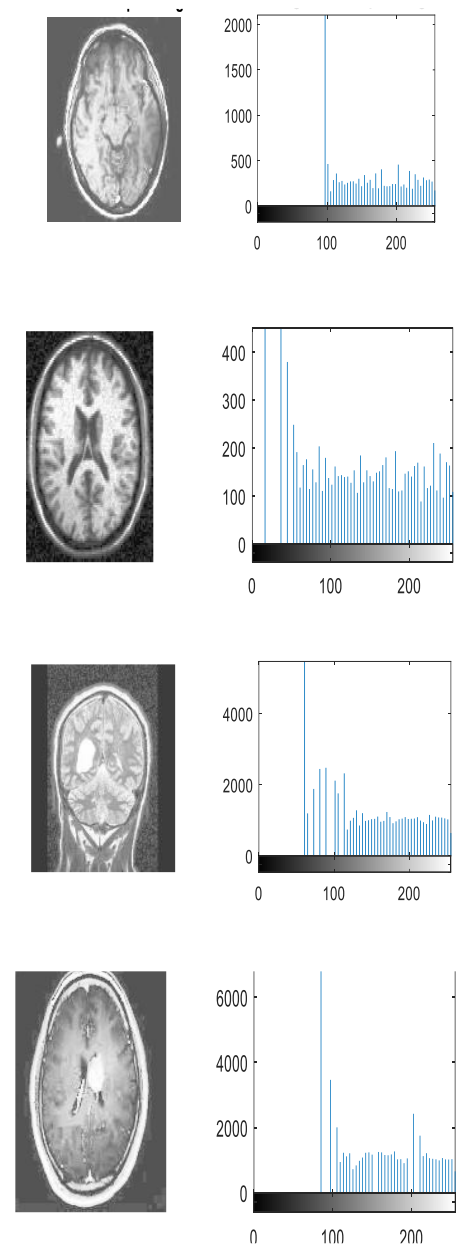

Fig. 2. First column shows the original input medical images and its Histogram output. Second column shows the enhanced output w.r.t input image and its histogram output. 
Simulation Results of AGCWD image enhancement and image segmentation using ACM with SBGFRLS, ORACM
AGCWD
ACM with SBGFRLS
ORACM output
output
Column (a)
Column (b)
Column (c)
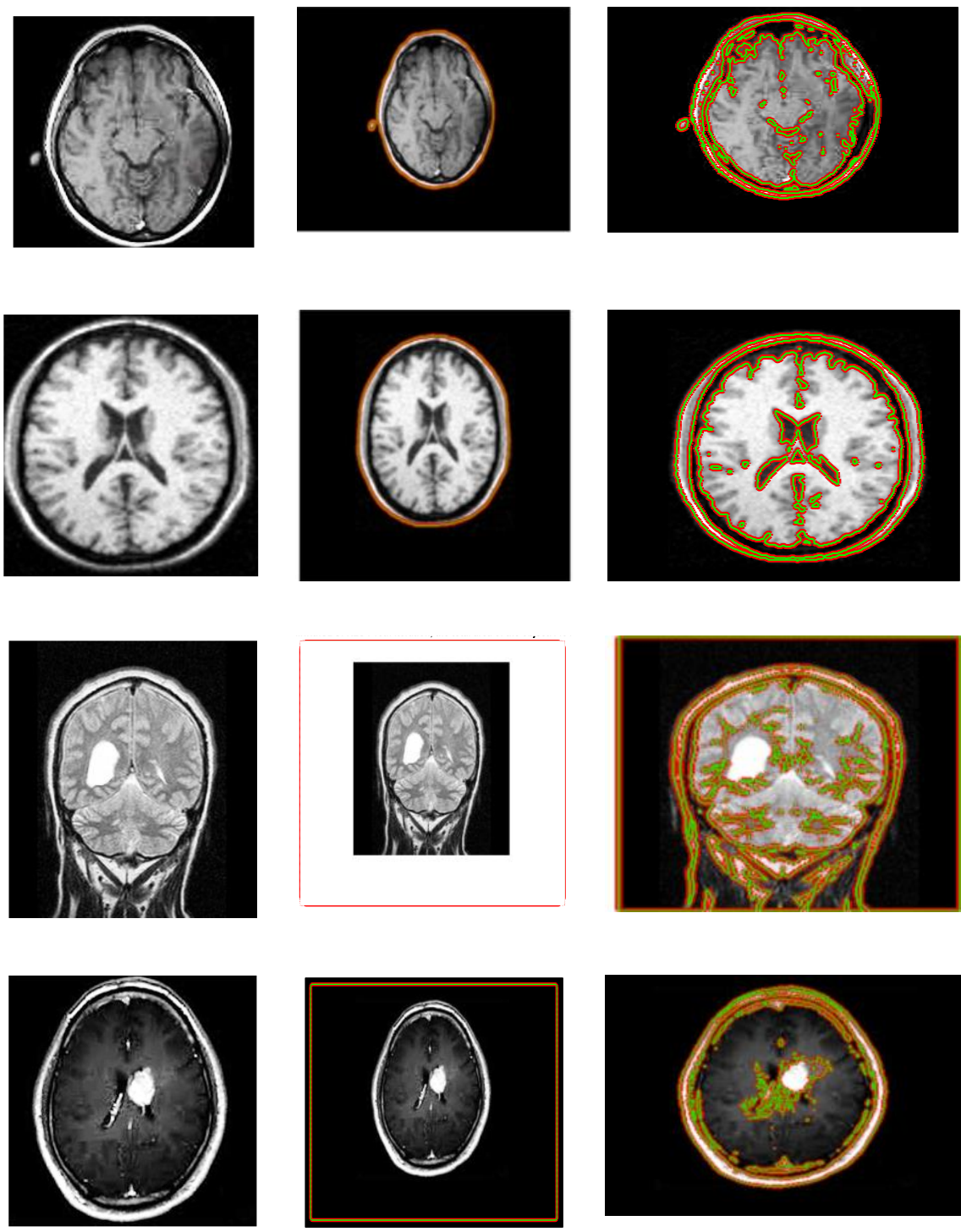

Fig. 3. First column depicts the AGCWD output. Second column depicts the ACM with SBGFRLS output w.r.t AGCWD output. Third Column depicts the ORACM output w.r.t AGCWD output. 
Table 1. Performance Metrics of Adaptive Gamma Correction Weighted Distribution based ORACM and ACM with SBGFRLS

\begin{tabular}{|l|c|c|c|c|c|c|}
\hline \multicolumn{1}{|c|}{ Images } & \multicolumn{2}{|c|}{ AGCWD based ACM with SBGFRLS } & \multicolumn{3}{c|}{ AGCWD based ORCAM } \\
\hline & Iterations & CPU time & $\begin{array}{c}\text { Total Area Cov- } \\
\text { ered }(\mathbf{m m} \mathbf{2})\end{array}$ & Iterations & CPU time & $\begin{array}{c}\text { Total Area Cov- } \\
\text { ered }(\boldsymbol{m m} \text { ) })\end{array}$ \\
\hline Image1 & 126 & $88.39 \mathrm{~s}$ & 24316 & 5 & $2.79 \mathrm{~s}$ & 30883 \\
\hline Image2 & 87 & $55.68 \mathrm{~s}$ & 35149 & 5 & $2.96 \mathrm{~s}$ & 45872 \\
\hline Image3 & 15 & $8.244 \mathrm{~s}$ & 103987 & 6 & $4.00 \mathrm{~s}$ & 145432 \\
\hline Image4 & 2 & $1.40 \mathrm{~s}$ & 9642 & 12 & $6.68 \mathrm{~s}$ & 131216 \\
\hline
\end{tabular}

Table 2. Performance Metrics of AGCWD algorithm for contrast enhancement

\begin{tabular}{|l|l|l|l|}
\hline \multicolumn{1}{|c|}{ Image 1 } & \multicolumn{1}{c|}{ Image 2 } & \multicolumn{1}{c|}{ Image 3 } & \multicolumn{1}{c|}{ Image 4 } \\
\hline $\mathrm{PSNR}=13.9532$ & $\mathrm{PSNR}=11.2460$ & $\mathrm{PSNR}=14.2706$ & $\mathrm{PSNR}=16.8362$ \\
\hline $\mathrm{MSE}=2616.7$ & $\mathrm{MSE}=4880.7$ & $\mathrm{MSE}=2432.3$ & $\mathrm{MSE}=1347.3$ \\
\hline
\end{tabular}

\section{Conclusion}

Methods for contrast enhancement established on histogram equalization are commonly applied. To smooth down the histogram weighted process is applied. In order to rise the brightness of an image additionally gamma correction is also taken into consideration. The work improves the AGCWD method that had implemented Gamma Correction as well as Weighted Distribution methods that helps in better enhancement of images during pre-processing of an image. Later in post-processing to an image ORACM level set method is applied on AGCWD for segmentation and is compared with ACM with SBGFRLS.

\section{References}

[1] Patel S., Bharath K.P., Balaji S., Muthu R.K. (2020) Comparative Study on Histogram Equalization Techniques for Medical Image Enhancement. In: Das K., Bansal J., Deep K., Nagar A., Pathipooranam P., Naidu R. (eds) Soft Computing for Problem Solving. Advances in Intelligent Systems and Computing, vol 1048. Springer, Singapore https://doi.org/10. 1007/978-981-15-0035-0 54

[2] S. H. Gangolli, A. Johnson Luke Fonseca and R. Sonkusare, "Image Enhancement using Various Histogram Equalization Techniques," 2019 Global Conference for Advancement in Technology (GCAT), , India, 2019, pp. 1-5, https://doi.org/10.1109/gcat 47503.2019.8978413.

[3] Somal S. (2020) Image Enhancement Using Local and Global Histogram Equalization Technique and Their Comparison. In: Luhach A., Kosa J., Poonia R., Gao XZ., Singh D. (eds) First International Conference on Sustainable Technologies for Computational Intelligence. Advances in Intelligent Systems and Computing, vol 1045. Springer, Singapore https://doi. org/10.1007/978-981-15-0029-9 58

[4] Sengupta S., Negi A. (2020) Comparative Analysis of Contrast Enhancement Techniques for MRI Images. In: Pandian A., Palanisamy R., Ntalianis K. (eds) Proceeding of the International Conference on Computer Networks, Big Data and IoT (ICCBI - 2019). ICCBI 2019. 
Lecture Notes on Data Engineering and Communications Technologies, vol 49. Springer, Cham. https://doi.org/10.1007/978-3-030-43192-1

[5] Pooja Patel, Arpana Bhandari (2019) A Review on Image Contrast Enhancement Techniques, IJO-Science (International Journal Online of Science) issn 2455-0108 vol. 5, issue 7, July 2019

[6] A. Kumar, S. V. Raghavendra Kommuri, H. Singh, A. Kumar and L. K. Balyan, Piecewise Gamma Corrected Weighted Framework for Fuzzified Dynamic Intensity Equalization for Optimal Image Enhancement, 2019 International Conference on Communication and Signal Processing (ICCSP), Chennai, India, 2019, pp. 0480-0484, https://doi.org/10.1109/iccsp. $\underline{2019.8697947}$

[7] Magudeeswaran Veluchamy, Bharath Subramani (2019) "Image contrast and color enhancement using adaptive gamma correction and histogram equalization", Optik (Elsevier) Vol: 183, April 2019, Pages 329-337. https://doi.org/10.1016/j.ijleo.2019.02.054

[8] Shih-Chia Huang, Fan-Chieh Cheng, Yi-Sheng Chiu, "Efficient Contrast Enhancement using Adaptive Gamma Correction with Weighting Distribution," IEE histogram equalization E.Trans. Image Process. VOL.No.22; pp.1032-1041. IEEE Transactions on Image Processing, Volume. 22, NO. 3, March 2013 https://doi.org/10.1109/tip.2012.2226047

[9] Zhuang, L.; Guan, Y. Image Enhancement Using Modified Histogram and Log-Exp Transformation. MDPI and ACS Style Symmetry 2019, 11, 1062. https://doi.org/10.3390/sym $\underline{11081062}$

[10] Fang, L., Qiu, T., Zhao, H. et al. A hybrid active contour model based on global and local information for medical image segmentation. Multidim Syst Sign Process 30, 689-703 (2019). https://doi.org/10.1007/s11045-018-0578-0

[11] Chinegaram K., Ramudu K., Srinivas A., Reddy G.R. (2020) Optimized Segmentation of Oil Spills from SAR Images Using Adaptive Fuzzy K-Means Level Set Formulation. In: Saini H., Singh R., Tariq Beg M., Sahambi J. (eds) Innovations in Electronics and Communication Engineering. Lecture Notes in Networks and Systems, vol 107. Springer, Singapore https://doi.org/10.1007/978-981-15-3172-9_40

[12] Ch.Kalyani, Ramudu Kama and Ganta Raghotham Reddy, “A review on optimized K-means and FCM clustering techniques for biomedical image segmentation using level set formulation" in Biomedical Research (India)-An International Journal of Medical Sciences, volume.29,No.20, 2018, (ISSN: 0970-938X), pp: 3660-3668. https://doi.org/10.4066/biomedicalresearch.29-18-1052

[13] Ramudu Kama, Kalyani Chinegaram , Ranga Babu Tummala and Raghotham Reddy Ganta Segmentation of Soft Tissues and Tumors from Biomedical Images using Optimized KMeans Clustering via Level Set formulation, I.J. Intelligent Systems and Applications, 2019, 9, 18-28. https://doi.org/10.5815/ijisa.2019.09.03

[14] J. Fang, H. Liu, L. Zhang, J. Liu and H. Liu, "Active Contour Driven by Weighted Hybrid Signed Pressure Force for Image Segmentation," in IEEE Access, vol. 7, pp. 97492-97504, 2019, https://doi.org/10.1109/access.2019.2929659.

[15] AnjaliWadhwa, AnujBhardwaj, VivekSingh Verma, A review on brain tumor segmentation of MRI images Magnetic Resonance Imaging, Volume 61, September 2019, Pages 247-259, https://doi.org/10.1016/j.mri.2019.05.043

[16] S. Osher, J. A. Sethian, "Fronts propagating with curvature-dependent speed: algorithms based on Hamilton-Jacobi formulations", J. Computational Physics, 79(1):12-49, 1988. https://doi.org/10.1016/0021-9991(88)90002-2 


\section{$7 \quad$ Authors}

Ch. Kalyani received his Bachelor of Engineering degree in Electronics \& Communications from Vaagdevi Engineering College, Warangal, JNTUH. She obtained her M. Tech in Digital Electronics and computer Engineering from, BITS, Warangal. Currently she is Assistant Professor in the department of ECE at Kamala Institute of Technology and Science, Singapur, Huzurabad, Telangana, India. Her Research Interest is Biomedical Image Processing and He published 4 International Journals and Conference till date in the field of Image Processing. kalyanichinegaram@gmail.com

Dr. Ramudu Kama received his Bachelor of Engineering degree in Electronics \& Communications from V. R. Sidhartha Engineering College, Vijayawada, AcharyaNagarjuna University, Guntur. He obtained his M. Tech in Digital Communication Engineering from, Kakatiya University, Warangal. He obtained his $\mathrm{PhD}$ degree from Acharya Nagarjuna University, Guntur, Andhra Pradesh, India Currently he is Assistant Professor in the department of ECE at Kakatiya Institute of Technology and Science, Warangal, Telangana, INDIA. His Research Interest is Biomedical Image Processing and He published 28 International Journals and Conference till date in the field of Image Processing. He is a Member of IETE, and ISTE.

Dr. Ganta Raghotham Reddy received his Bachelor of engineering degree in Electronics \& Communications from Gulbarga University; He obtained his M. Tech in Digital Communication Engineering from Maulana Azad National Institute of Technology (MANIT), Bhopal; He obtained his PhD degree from Osmania University, Hyderabad, Telangana, India. Currently he is Professor in the department of Electronics and Communication Engineering, Kakatiya Institute of Technology and Science (KITS), Warangal, A.P. India. His areas of research include Image and Signal processing. Member of IETE, ISTE and IEEE. grrece9@gmail.com

Article submitted 2020-09-13. Resubmitted 2020-10-02. Final acceptance 2020-10-05. Final version published as submitted by the authors. 